\title{
Function-Preserving Surgery in Gastric Cancer
}

\author{
Jan Andrew D. Bueno, M.D. ${ }^{1,2}$, Young-Suk Park, M.D. ${ }^{1}$, Sang-Hoon Ahn, M.D. ', Do Joong Park, M.D., Ph.D. ', \\ Hyung-Ho Kim, M.D., Ph.D. ${ }^{1}$ \\ 'Department of Surgery, Seoul National University Bundang Hospital, Seoul National University College of Medicine, Seongnam, Korea, ${ }^{2}$ Department of \\ Surgery, University of Santo Tomas Hospital, Philippines
}

\begin{abstract}
The rising incidence of early gastric cancer has enabled the development of function-preserving gastrectomy with the focus on post gastrectomy quality of life and adherence to sound oncologic principles. It is concurrent with the growing popularity of minimally invasive surgery; and both are commonly used together. The different kinds of function-preserving gastrectomy included in this review are: pylorus-preserving and proximal gastrectomy, vagus nerve preservation, sentinel node navigation, and various endoscopic \& minimally-invasive techniques. In this article the indications, techniques, oncologic safety, functional benefit, and outcomes of each kind of function-preserving gastrectomy are discussed.
\end{abstract}

Keywords: Function-preserving gastrectomy (FPG), Early gastric cancer (EGC), Pylorus-preserving gastrectomy (PPG), Proximal gastrectomy (PG), Laparoscopic gastrectomy
Received July 6, 2018

Revised September 11, 2018

Accepted September 12, 2018

Corresponding author

Do Joong Park

Department of Surgery, Seoul National

University Bundang Hospital, Seoul

National University College of

Medicine, 166 Gumi-ro, Bundang-gu,

Seongnam 13620, Korea

Tel: +82-31-787-7097

Fax: +82-31-787-4078

E-mail: djpark@snubh.org

ORCID:

http://orcid.org/0000-0001-9644-6127

Copyright $\odot 2018$ The Journal of Minimally Invasive Surgery. All rights reserved.
This is an Open Access article distributed under the terms of the Creative Commons Attribution Non-Commercial License (http:// creativecommons.org/licenses/by-nc/4.0/) which permits unrestricted non-commercial use, distribution, and reproduction in any medium, provided the original work is properly cited.

\section{INTRODUCTION}

The proportion of early gastric cancer (EGC) in East Asia has been increasing due to the implementation of screening programs in South Korea and Japan. In 2014, Stage I gastric cancers comprised $63.9 \%$ of all patients who received surgical treatment for gastric cancer in South Korea. ${ }^{1}$ This made the prognosis for majority of gastric cancer patients favorable and thus the shift of focus on quality of life post gastrectomy is inevitable.

Function-preserving gastrectomy (FPG) was developed to prevent post-gastrectomy syndromes experienced after conventional radical gastrectomy. It is characterized by a less radical but curative resection that maintains the reservoir and mixing/grinding functions of the stomach, as well as its endocrine and metabolic function. It is concurrent with the popularity of laparoscopic surgery and thus are used in combination in some centers.

In this review article, the various types of FPG will be discussed which are: pylorus-preserving gastrectomy, proximal gastrectomy, vagus nerve-preserving gastrectomy, sentinel node navigation surgery, and endoscopic resection (EMR, ESD, LECS, CLEAN-NET, NEWS).

\section{PYLORUS-PRESERVING GASTRECTOMY (PPG)}

PPG was initially developed by Maki et al. for the treatment of benign ulcers in the middle third of the stomach and has then been adapted for treatment in EGC. Essential features of 
the procedure include: preservation of the sphincteric function of the pylorus and retaining of the storage function of the prepyloric antrum. With this technique, the physiological gastric emptying is theoretically maintained; hence, alkaline reflux, dumping syndrome, disturbed bowel habits, and weight loss may be reduced in contrast to the standard distal gastrectomy (DG) with Billroth I reconstruction. ${ }^{2}$

According to the Japanese gastric cancer treatment guidelines, ${ }^{3}$ PPG can be considered for the treatment of clinical T1 N0 M0 gastric cancers in the middle portion of the stomach with distal tumor border at least $4 \mathrm{~cm}$ proximal to the pylorus.

The standard technique of pylorus preservation is comprised of preservation of the hepatic branch of the vagus nerve and the infra-pyloric vessels, with some minor variation among surgeons. ${ }^{4}$ This maintains the innervation, blood supply, and venous drainage of the pylorus.

$\mathrm{D} 1 / \mathrm{D} 1+$ lymphadenectomy is recommended for cT1N0 gastric cancer patients according to the Japanese Gastric Cancer Guidelines, which for PPG, comprises dissection of lymph nodes station $1,3,4 \mathrm{sb}, 4 \mathrm{sd}, 6, \& 7$ for D1 and inclusion of $8 \mathrm{a}$ \& 9 for D1+. ${ }^{3}$ Suprapyloric lymph node dissection is omitted to preserve the right gastric vessels and hepatic branch of the vagus, which has been considered a pitfall of PPG. ${ }^{5}$ However, a Japanese study reported a $0.2 \%$ rate of metastases to the suprapyloric lymph nodes in $\mathrm{T} 1$ cancers in the middle onethird of the stomach. ${ }^{6}$ CT scan and endoscopic ultrasound are commonly used pre-operatively to ascertain accuracy of the diagnosis of cT1N0 cases who are candidates for PPG.

\section{Outcomes}

In a study using quality-of-life questionnaires PPG was found-out to have significantly lower scores in diarrhea and dumping syndrome compared to DG. ${ }^{7}$ A meta-analysis ${ }^{8}$ involving 1,213 patients found PPG to be a preventive factor of dumping syndrome, bile reflux, gastritits, and gallstone formation, while proving to be beneficial in weight regain.

Delayed gastric emptying is one of the most common complications after PPG. Incidence of gastric stasis was 6.2\% 7.8\% in patients who underwent laparoscopy-assisted PPG. ${ }^{5}$ Postoperative edema and neurologic dysfunction have been theorized to cause such complication, therefore preservation of the vagal innervation and right gastric \& infrapyloric veins for blood flow drainage are advocated in PPG. ${ }^{9}$ Nakane et al. ${ }^{10}$ reported a $35 \%$ incidence in delayed gastric emptying in patients with a $1.5 \mathrm{~cm}$ antral cuff length vs. $10 \%$ in patients with a $2.5 \mathrm{~cm}$ antral cuff length. The optimum antral cuff length is not yet established, but has tended to become longer in more recent studies. ${ }^{5}$

The 5-year survival rate of patients who underwent PPG was $98 \%$ in a study by Hiki et al. ${ }^{11}$ Comparing laparoscopyassisted PPG to laparoscopy-assisted DG, Suh et al. ${ }^{12}$ reported a similar 3-year recurrence-free survival rate of $98.2 \%$ vs $98.8 \%$, respectively. A meta-analysis by Xiao et al. ${ }^{8}$ showed a similar 5-year survival rate of PPG vs DG.

\section{Laparoscopy in PPG}

The use of laparoscopy in function-preserving gastrectomy has gained interest and popularity in the recent years. Laparoscopy-assisted PPG has been established as a feasible and safe procedure with reduced blood loss, pain, and faster recovery compared with open PPG. ${ }^{13}$ Anastomosis can be easily performed from a small upper abdominal incision directly above the anastomosis site (Fig. 1).

A multicenter randomized controlled trial comparing laparoscopic PPG vs laparoscopic DG (KLASS 04-Korean laparoscopic Gastrointestinal Surgery Study) is on-going in South Korea. It will primarily investigate the incidence of dumping syndrome, as well as the relapse-free \& overall survival, morbidity, mortality, QOL, and other secondary outcome measures.

Totally laparoscopic PPG has also been reported, although a longer operative time is required compared to laparoscopyassisted PPG. ${ }^{14}$ Anastomosis is done using intracorporeal delta-shaped anastomosis and a combination of stapler \& handsewn procedures. ${ }^{2}$ Confirming the tumor location by use of pre-operative placement of marking clips and intraoperative gastroscopy has ensured the oncologic safety of this procedure.

Robotic gastrectomy has not been proven to be superior than laparoscopic gastrectomy. ${ }^{15}$ Although it may provide

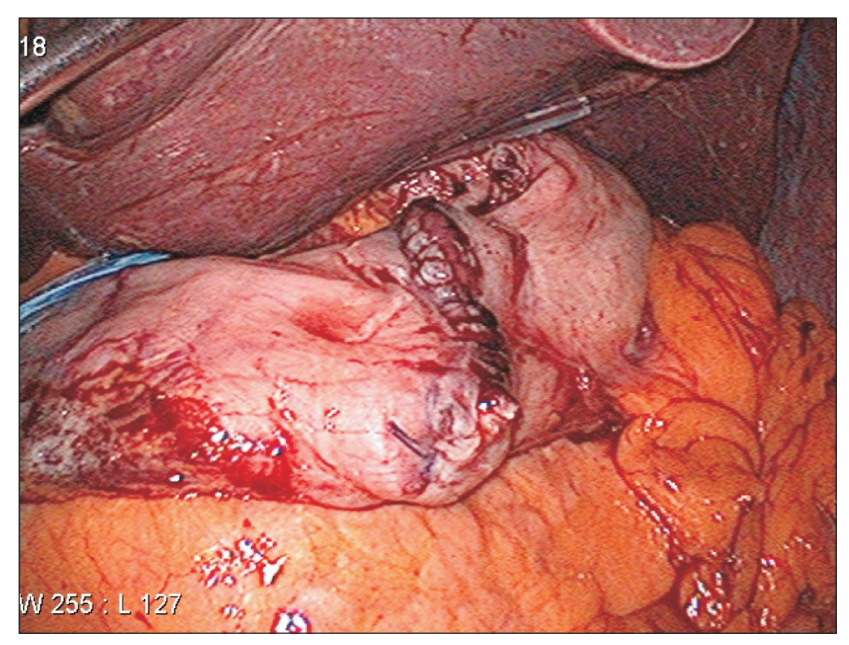

Fig. 1. Gastro-gastric anastomosis after laparoscopic pylorus-preserving gastrectomy. 
a superior operating environment, as it eliminates surgeon tremor and provides a highly magnified imaging with a steady fixed camera. ${ }^{5}$ Robot assisted PPG has been reported but has not shown any difference in terms of patient outcomes when compared to laparoscopy assisted PPG. ${ }^{16}$

\section{PROXIMAL GASTRECTOMY (PG)}

The incidence of cancer in the upper third of the stomach is steadily rising. In 2014, there were 2,365 (16\%) cases of cancers in the upper third of the stomach treated with surgery in South Korea, a $2.6 \%$ increase from 2009. In congruence with the growth of the proportion of EGC, laparoscopic PG is the best theoretical treatment option for proximal EGC and outweighs open PG, open total gastrectomy (TG), and laparoscopic TG. ${ }^{17}$ Quality of life may be improved with PG due to the preservation of the function of the distal stomach along with the pyloric ring. ${ }^{9}$ However, $\mathrm{PG}$ is not a popular surgical choice, with only $1.1 \%$ (168) of all gastrectomy cases done in South Korea at 2014. This is probably due to three main concerns with PG: functional benefits, oncological safety, and anastomosis-related complications. These will be discussed in the succeeding text.

The Japanese gastric cancer treatment guidelines 2014 (ver 4) recommends that PG can be considered for cT1N0 proximal gastric tumors where more than half of the distal stomach can be preserved; and D1/D1+ lymphadenectomy for cT1N0 tumors comprising lymph nodes 1, 2, 3a, 4sa, 4sb, \& 7 for D1; with the inclusion of 8a, 9, \& 11p for D1+. ${ }^{3}$ The distal stomach is preserved along with its blood supply (right gastric and right gastroepiploic vessels) and innervation (distal branches of the vagus nerve).

\section{Outcomes}

Many studies report conflicting benefit of PG compared to TG in terms of nutrition status (serum protein, albumin, and cholesterol), body weight, and anemia. ${ }^{18-21}$ However, longterm follow-up data have shown that hemoglobin levels in PG are significantly higher than in TG, which is reflected in the lower requirement for vitamin $\mathrm{B}_{12}$ supplementation after $\mathrm{PG}$ compared to TG. ${ }^{22}$ Takiguchi et al. ${ }^{23}$ reported improved quality of life in terms of weight loss, necessity of additional meals, diarrhea, and dumping in patients who underwent open PG vs open TG.

The oncologic safety of PG is questioned due to the omission of dissection of lymph node stations 5 and 6 plus the potential of remnant stomach cancer. However, lymph node metastasis in EGC are estimated to be at 10 20\%. Additionally, Kong et al. ${ }^{24}$ reported that proximal EGC only metastasize to
LN stations 2, 3, and 7. And Kitamura et al. ${ }^{25}$ did not observe LN metastasis on the lower stomach among proximal gastric cancers confined to the muscularis propia (T2).

In a meta-analysis comprising 1,364 patients, the 5-year survival rate in PG was not statistically different with TG (60.9\% vs 64.4\%). But recurrence is expectedly higher in PG compared to TG $(38.7 \%$ vs $24.4 \%){ }^{26}$

Remnant gastric cancer is higher after open PG (3.6 9.1\%) than after open DG (0.4 2.5\%). Endoscopic surveillance after surgery is an important tool for detection of recurrence. Although intubation of the endoscope can be difficult in PG with an esophagojejunostomy (EJ) reconstruction, especially with a greater than $10 \mathrm{~cm}$ interposed jejunal length. ${ }^{27}$

Esophagogastrostomy (EG) and EJ are the most frequently used reconstruction technique for PG. EG only involves one anastomosis, lesser operative time, and blood loss but has a higher frequency of postoperative reflux esophagitis and anastomotic stricture. Various modifications have been tried (gastropexy, fundoplication, gastric tube, lower esophageal sphincter preserving, etc.) but the anastomosis-related late complications were significantly higher in EG after PG compared to Roux-en-Y EJ after TG in various studies (27.4 67.4\% vs 7.4 8.7\%). $18,19,26,28$

EJ has been used to prevent the anastomosis-related complications of EG. Jejunal interposition, jejunal pouch, and double tract reconstruction (DTR) are among the EJ procedures done. EJ after PG is similar to Roux-en-Y EJ after TG in terms of anastomosis-related late complications ( $0 \sim 10.2 \%$ vs $1.8 \sim 8.7 \%)^{18,29-31}$ Although abdominal fullness, discomfort rumbling, and hiccups are more frequently found after EJ in PG due to the disturbance of food passage by the interposed bowel. $^{9}$

\section{Laparoscopy in PG}

Few studies have compared laparoscopic PG to open PG. Kinoshita et $a .^{32}$ reported that laparoscopic PG has a longer operative time but decreased blood loss, and similar complication rates compared to open PG. The most important technical challenge in laparoscopic PG is the reconstruction method, which is still not well established. ${ }^{33} \mathrm{Ahn}$ et al. ${ }^{33}$ reported a case series on laparoscopic PG using DTR and showed that it is a feasible, simple, and useful reconstruction method with excellent postoperative outcomes in terms of reflux symptoms (Fig. 2).

An on-going multicenter, prospective Phase III trial (KLASS 05) in South Korea aims to compare laparoscopic PG with DTR and laparoscopic TG, with hemoglobin change at 2 years post-gastrectomy and vitamin B12 cumulative supplement quantity as the primary endpoint. 

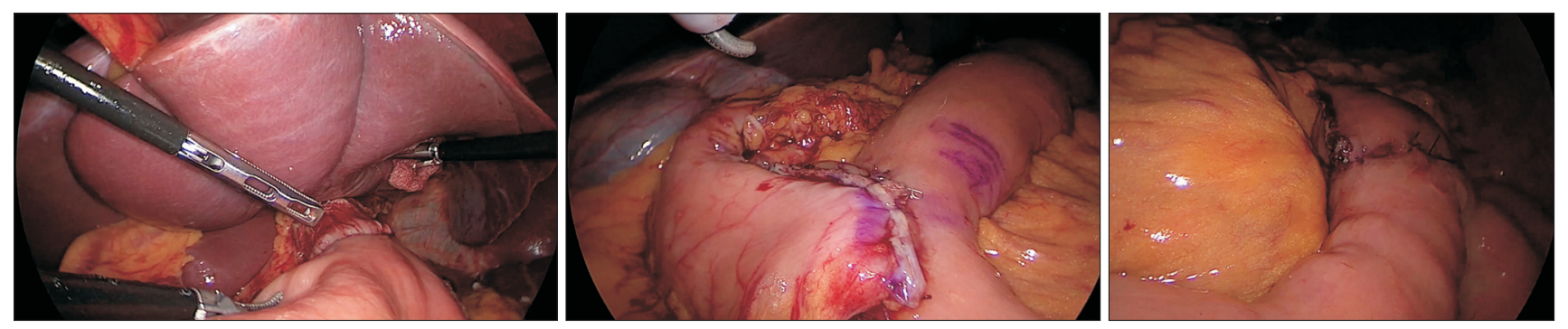

Fig. 2. Laparoscopic proximal gastrectomy with double tract reconstruction which comprises esophago-jejunostomy, gastro-jejunostomy and jejunojejunostomy.

Single-port laparoscopic PG with DTR has been reported by Lee et al..$^{34}$ Despite the technical challenge caused by poor ergonomics, it provided cosmetic benefit and a good quality of life for the patient. The use of Robotics in a few cases of PG has been reported as part of general use of robotics in gastrectomy. ${ }^{15}$ There are no specific reports on the benefit of robotics in $\mathrm{PG}$.

\section{VAGUS NERVE-PRESERVING GASTRECTOMY (VNPG)}

In conventional D2 gastrectomy for gastric cancer, the celiac and hepatic branches of the vagus are removed en bloc with the left gastric artery and the whole of the lesser omentum. ${ }^{35}$ Vagus nerve preservation in open gastrectomy was introduced in 1991 at Japan, for early gastric cancer cases to decrease postgastrectomy syndrome. In this procedure, the hepatic branches of the anterior vagal trunk that innervates the liver and biliary tract are preserved, as well as the celiac branches of the posterior vagal trunk which innervates the small intestines. ${ }^{36}$ It is an integral part of PPG and has been described in numerous articles. But is also used in early gastric cancers with distal, total, and proximal gastrectomy. ${ }^{35}$

For preservation of the hepatic branch, the lesser omentum should be divided below the nerve. During dissection of the common hepatic artery lymph nodes, the hepatic nerve plexus should be identified and preserved. ${ }^{36}$ The posterior trunk of the vagus nerve and its branches lie within the gastropancreatic peritoneal fold. Ando et $a{ }^{35}{ }^{35}$ identified three types of variations in the course of the celiac branch depending on its relationship to the left gastric artery, and found that $81.8 \%$ had the celiac branch lay closer to the artery than to the crus. Preservation of the celiac branch entails meticulous dissection when isolating the left gastric artery (Fig. 3).

\section{Outcomes}

The benefits of VNPG are seen in the superior recovery

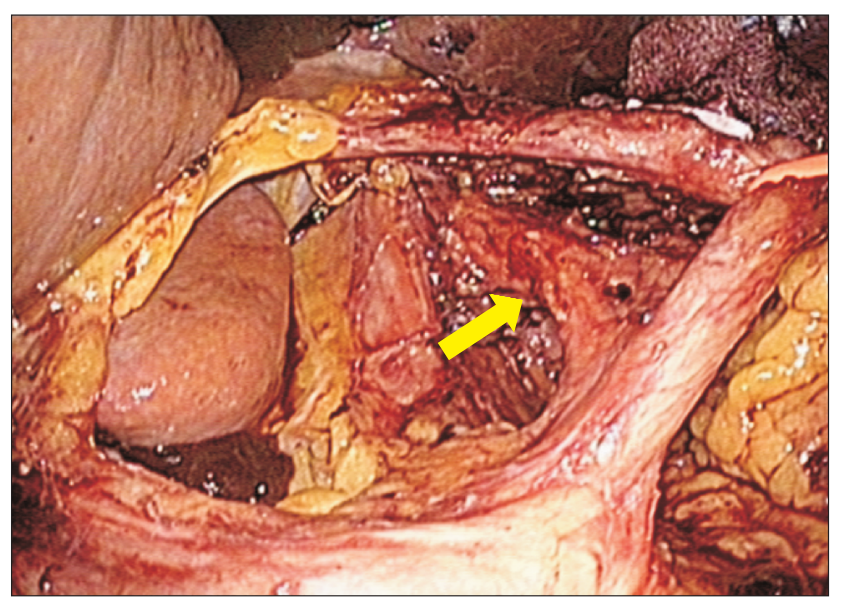

Fig. 3. Vagus nerve-preserving gastrectomy. Hepatic and celiac branches are identified and preserved during the surgery.

of body weight and reduction in the incidence of gallstone development. ${ }^{35}$ A randomized controlled trial ${ }^{37}$ in South Korea comparing vagus nerve-preserving distal gastrectomy to conventional gastrectomy showed significantly less diarrhea and appetite loss at 12 months with vagus nerve preservation. There was no difference in the long-term clinical complications, recurrence, and survival.

\section{Laparoscopy in VNPG}

Laparoscopic VNPG has been reported mostly in articles on laparoscopic PPG. Under the laparoscopic view, fine vessels and nerves are seen more clearly because of the magnification and thus might be more reliable than open techniques. ${ }^{36}$

\section{SENTINEL NODE NAVIGATION SURGERY (SNNS)}

The sentinel node (SN) is defined as the first lymph node to receive lymphatic drainage from the primary tumor, and lymph node metastasis is assumed to initially occur at this 
site $^{38}$ The principle of SNNS is to identify and check the SNs for metastasis so that excessive lymphadenectomy for nonpathologic nodes may be prevented and extent of gastric resection be minimized. This concept has been applied to breast cancer and melanoma treatment and has led to less morbidity and improved quality of life in patients. ${ }^{39-41}$

But due to the multidirectional lymphatic drainage of the stomach and the possibility of skip metastasis, the application of SNNS in gastric cancer has been a challenge. ${ }^{38}$ Other issues with SNNS is the use of the appropriate tracer, how to identify \& examine the SNs, options for primary tumor control, and which subgroup of patients will SNNS be applicable for. ${ }^{42}$

A combination of dye-based and radioisotope-based techniques are the most commonly used method for sentinel node mapping and identification. Peritumoral submucosal endoscopic injection of the dye is usually done and infrared fluorescence imaging of the surgical field has been used for better visualization. Hematoxylin and eosin has been the most commonly used technique for intraoperative diagnosis of metastasis in sentinel nodes and accuracy ranges from 74 100\% in the literature. ${ }^{42}$ Other techniques include immunohistological staining and reverse transcriptase polymerase chain reaction, which seem promising especially with concerns on micrometastasis. ${ }^{42}$ For primary tumor control; the other function preserving gastrectomy methods such as PG \& PPG have been used, as well as less extensive resections such as wedge resection, segmental resection, and various endoscopic fullthickness \& local resection techniques, ${ }^{43}$ however there is no consensus on the optimal method. ${ }^{44}$ The studies from East Asia have used SNNS mostly in T1 and some in T2 tumors. Some Western studies included up to T3 tumors. ${ }^{42}$

The Japanese Society of Sentinel Node Navigation Surgery ${ }^{45}$ analyzed 397 patients with cT1-2N0M0 gastric cancers with $<4$ $\mathrm{cm}$ tumor size. They perfomed SN mapping and identification followed by standard gastrectomy with D2 lymphadenectomy. The detection rate, sensitivity, and accuracy of SN examination were 97.5\% (387/397), 93\% (53/57), and 99\% (383/387), respectively. False-negative rate was only $1 \%$ (four patients). Their work also suggested that lymphatic basin based surgery (sentinel lymph node and downstream lymphatic flow) may be recommended due to findings of metastatic nodes in only the lymphatic basin of some cases with negative SNs.

The SENORITA trial (SEntinel Node ORIented Tailored Approach) in South Korea will evaluate stomach preserving surgery with sentinel basin dissection versus standard gastrectomy in terms of disease-free survival, morbidity, mortality, and quality of life in patients with early gastric cancer. ${ }^{38,44}$

\section{ENDOSCOPIC RESECTION}

Endoscopic resection is considered for tumors that have a very low possibility of lymph node metastasis and are suitable for en-bloc resection. Some are used as primary tumor control for SNNS. The two most frequently used methods of endoscopic resection are Endoscopic mucosal resection (EMR) and Endoscopic submucosal dissection (ESD). The Japanese gastric cancer treatment guidelines ${ }^{3}$ state that EMR or ESD is indicated as a standard treatment for gastric adenocarcinoma of the differentiated-type without ulcerative findings, of which the depth of invasion is clinically diagnosed as Tla and the diameter is $\leq 2 \mathrm{~cm}$. Expanded indications have also been described but are considered investigational.

In EMR, the lesion, together with the surrounding mucosa, is lifted by submucosal injection of saline and removed using a high-frequency steel snare. ${ }^{3}$ A major limitation of EMR is incomplete resection of lesions $>2 \mathrm{~cm}$ due to size limitation of the instruments used. ${ }^{46}$

While in ESD, the mucosa surrounding the lesion is circumferentially incised using a high-frequency electric knife and the submucosal layer is dissected from the proper muscle layer. ${ }^{3}$ The size limitation in EMR is addressed by ESD and is considered superior to EMR because it enables precise pathologic T staging for large EGC. ${ }^{46}$ In a recent metaanalysis, ${ }^{47}$ ESD was shown to have advantages in en bloc resection, histologically complete resection, and local recurrence rate compared to EMR. Although mean operation time and complications such as perforation was higher in the ESD technique.

Hahn et $\mathrm{all}^{48}$ found that ESD had lower acute complication rates compared to surgery $(8.1 \%$ vs $18.1 \%)$ but had similar 5 year-overall survival (96.4\% vs 97.2\%) and disease-specific survival rate (99.6\% vs $99.2 \%)$. However, the annual incidence of recurrence was $2.18 \%$ in ESD and $0.19 \%$ in the surgery group.

Hybrid surgery or the concept of laparoscopic and endoscopic cooperative surgery (LECS) for early gastric cancer has been reported. Endoscopic full-thickness resection (EFTR) is usually done with laparoscopic assistance to facilitate safe resection of the lesion, repair of the ensuing defect, and even limited lymph node dissection. ${ }^{49}$ Dissection is done intraluminally with submucosal injection and circumferential endoscopic mucosal incision, followed by intentional perforation to facilitate complete full thickness resection under laparoscopic countertraction. ${ }^{50}$ Iatrogenic tumor seeding is a concern with this procedure since there is a possibility of tumor cells in the gastric juice to spread by spillage or by contact of exposed surface of the lesion with the peritoneum or laparoscopic instruments. ${ }^{49}$

Non-exposure techniques for full-thickness resection have 
been developed to prevent iatrogenic seeding such as wedge resection, combination of laparoscopic and endoscopic approaches to neoplasia with non-exposure technique (CLEANNET), and non-exposed endoscopic wall-inversion surgery (NEWS). ${ }^{49}$

\section{CONCLUSION}

Function-preserving gastrectomy has revolutionized treatment for early gastric cancer. The more popular types and techniques have been discussed in this review article and quite a few more are currently being developed with the advancement of skills \& technology. There are important matters that have to be addressed in function-preserving gastrectomy, which are: oncologic safety, actual functional benefit for patients, proper selection of patients, availability and expertise in the use of new technique and equipment including laparoscopy and robotics.

\section{REFERENCES}

1) Information Committee of Korean Gastric Cancer Association. Korean Gastric Cancer Association Nationwide Survey on Gastric Cancer in 2014. J Gastric Cancer 2016;16:131-140.

2) Nunobe S, Hiki N. Function-preserving surgery for gastric cancer: current status and future perspectives. Transl Gastroenterol Hepatol 2017;2:77.

3) Japanese Gastric Cancer Association. Japanese gastric cancer treatment guidelines 2014 (ver. 4). Gastric Cancer 2017;20:1-19.

4) Shibata C, Saijo F, Kakyo M, et al. Current status of pyloruspreserving gastrectomy for the treatment of gastric cancer: a questionnaire survey and review of literatures. World J Surg 2012;36: 858-863.

5) Oh SY, Lee HJ, Yang HK. Pylorus-Preserving Gastrectomy for Gastric Cancer. J Gastric Cancer 2016;16:63-71.

6) Nakajima T, Yamaguchi T. Gastric Cancer Data Base in Cancer Institute, Japan; 1946-2004. Tokyo: Kanehara \& Co, Ltd; 2006.

7) Fujita J, Takahashi M, Urushihara T, et al. Assessment of postoperative quality of life following pylorus-preserving gastrectomy and Billroth-I distal gastrectomy in gastric cancer patients: results of the nationwide postgastrectomy syndrome assessment study. Gastric Cancer 2016;19:302-311.

8) Xiao XM, Gaol C, Yin W, Yu WH, Qi F, Liu T. Pylorus-Preserving versus Distal Subtotal Gastrectomy for Surgical Treatment of Early Gastric Cancer: A Meta-Analysis. Hepatogastroenterology 2014;61:870-879.

9) Hiki N, Nunobe S, Kubota T, Jiang X. Function-preserving gastrectomy for early gastric cancer. Ann Surg Oncol 2013;20:26832692.

10) Nakane $Y$, Michiura T, Inoue $K$, Sato $M$, Nakai $K$, Yamamichi $K$.
Length of the antral segment in pylorus-preserving gastrectomy. Br J Surg 2002;89:220-224.

11) Hiki N, Sano T, Fukunaga T, Ohyama S, Tokunaga M, Yamaguchi T. Survival benefit of pylorus-preserving gastrectomy in early gastric cancer. J Am Coll Surg 2009;209:297-301.

12) Suh YS, Han DS, Kong SH, et al. Laparoscopy-assisted pyloruspreserving gastrectomy is better than laparoscopy-assisted distal gastrectomy for middle-third early gastric cancer. Ann Surg 2014; 259:485-493.

13) Nunobe S, Hiki N, Fukunaga $T$, et al. Laparoscopy-assisted pylorus-preserving gastrectomy: preservation of vagus nerve and infrapyloric blood flow induces less stasis. World J Surg 2007;31: 2335-2340.

14) Kumagai $K$, Hiki N, Nunobe $S$, et al. Totally laparoscopic pyloruspreserving gastrectomy for early gastric cancer in the middle stomach: technical report and surgical outcomes. Gastric Cancer 2015; 18:183-187.

15) Kim HI, Han SU, Yang HK, et al. Multicenter Prospective Comparative Study of Robotic Versus Laparoscopic Gastrectomy for Gastric Adenocarcinoma. Ann Surg 2016;263:103-109.

16) Han DS, Suh YS, Ahn HS, et al. Comparison of Surgical Outcomes of Robot-Assisted and Laparoscopy-Assisted Pylorus-Preserving Gastrectomy for Gastric Cancer: A Propensity Score Matching Analysis. Ann Surg Oncol 2015;22:2323-2328.

17) Jung DH, Ahn SH, Park DJ, Kim HH. Proximal Gastrectomy for Gastric Cancer. J Gastric Cancer 2015;15:77-86.

18) An JY, Youn HG, Choi MG, Noh JH, Sohn TS, Kim S. The difficult choice between total and proximal gastrectomy in proximal early gastric cancer. Am J Surg 2008;196:587-591.

19) Wen $L$, Chen $X Z$, Wu B, et al. Total vs. proximal gastrectomy for proximal gastric cancer: a systematic review and meta-analysis. Hepatogastroenterology 2012;59:633-640.

20) Kosuga T, Ichikawa D, Komatsu S, et al. Feasibility and Nutritional Benefits of Laparoscopic Proximal Gastrectomy for Early Gastric Cancer in the Upper Stomach. Ann Surg Oncol 2015;22 Suppl 3:S929-935.

21) Son MW, Kim YJ, Jeong GA, Cho GS, Lee MS. Long-Term Outcomes of Proximal Gastrectomy versus Total Gastrectomy for Upper-Third Gastric Cancer. J Gastric Cancer 2014;14:246-251.

22) Yoo CH, Sohn BH, Han WK, Pae WK. Proximal gastrectomy reconstructed by jejunal pouch interposition for upper third gastric cancer: prospective randomized study. World J Surg 2005;29: 1592-1599.

23) Takiguchi N, Takahashi M, Ikeda M, et al. Long-term qualityof-life comparison of total gastrectomy and proximal gastrectomy by postgastrectomy syndrome assessment scale (PGSAS-45): a nationwide multi-institutional study. Gastric Cancer 2015;18:407416.

24) Kong SH, Kim JW, Lee HJ, Kim WH, Lee KU, Yang HK. Reverse double-stapling end-to-end esophagogastrostomy in proximal 
gastrectomy. Dig Surg 2010;27:170-174.

25) Kitamura K, Yamaguchi T, Nishida S, et al. The operative indications for proximal gastrectomy in patients with gastric cancer in the upper third of the stomach. Surg Today 1997;27:993-998.

26) Pu YW, Gong W, Wu YY, Chen Q, He TF, Xing CG. Proximal gastrectomy versus total gastrectomy for proximal gastric carcinoma. A meta-analysis on postoperative complications, 5-year survival, and recurrence rate. Saudi Med J 2013;34:1223-1228.

27) Tokunaga M, Ohyama S, Hiki N, et al. Endoscopic evaluation of reflux esophagitis after proximal gastrectomy: comparison between esophagogastric anastomosis and jejunal interposition. World J Surg 2008;32:1473-1477.

28) Katsoulis IE, Robotis JF, Kouraklis G, Yannopoulos PA. What is the difference between proximal and total gastrectomy regarding postoperative bile reflux into the oesophagus? Dig Surg 2006;23: 325-330.

29) Zhao P, Xiao SM, Tang LC, Ding Z, Zhou X, Chen XD. Proximal gastrectomy with jejunal interposition and TGRY anastomosis for proximal gastric cancer. World J Gastroenterol 2014;20:8268-8273.

30) Katai H, Morita S, Saka M, Taniguchi H, Fukagawa T. Long-term outcome after proximal gastrectomy with jejunal interposition for suspected early cancer in the upper third of the stomach. Br J Surg 2010;97:558-562.

31) Nakamura M, Nakamori M, Ojima $T$, et al. Reconstruction after proximal gastrectomy for early gastric cancer in the upper third of the stomach: an analysis of our 13-year experience. Surgery 2014; 156:57-63.

32) Kinoshita T, Gotohda N, Kato Y, Takahashi S, Konishi M, Kinoshita T. Laparoscopic proximal gastrectomy with jejunal interposition for gastric cancer in the proximal third of the stomach: a retrospective comparison with open surgery. Surg Endosc 2013; 27:146-153.

33) Ahn SH, Jung DH, Son SY, Lee CM, Park DJ, Kim HH. Laparoscopic double-tract proximal gastrectomy for proximal early gastric cancer. Gastric Cancer 2014;17:562-570.

34) Lee CM, Park DW, Jung DH, et al. Single-Port Laparoscopic Proximal Gastrectomy with Double Tract Reconstruction for Early Gastric Cancer: Report of a Case. J Gastric Cancer 2016;16:200206.

35) Ando S, Tsuji H. Surgical technique of vagus nerve-preserving gastrectomy with D2 lymphadenectomy for gastric cancer. ANZ J Surg 2008;78:172-176.

36) Kojima K, Yamada H, Inokuchi M, Kawano T, Sugihara K. Functional evaluation after vagus-nerve-sparing laparoscopically assisted distal gastrectomy. Surg Endosc 2008;22:2003-2008.

37) Kim SM, Cho J, Kang D, et al. A Randomized Controlled Trial of Vagus Nerve-preserving Distal Gastrectomy Versus Conventional Distal Gastrectomy for Postoperative Quality of Life in Early Stage
Gastric Cancer Patients. Ann Surg 2016;263:1079-1084.

38) Park JY, Kim YW, Ryu KW, et al. Assessment of laparoscopic stomach preserving surgery with sentinel basin dissection versus standard gastrectomy with lymphadenectomy in early gastric cancer-A multicenter randomized phase III clinical trial (SENORITA trial) protocol. BMC Cancer 2016;16:340.

39) Valsecchi ME, Silbermins D, de Rosa N, Wong SL, Lyman GH. Lymphatic mapping and sentinel lymph node biopsy in patients with melanoma: a meta-analysis. J Clin Oncol 2011;29:14791487.

40) Veronesi U, Paganelli G, Viale G, et al. A randomized comparison of sentinel-node biopsy with routine axillary dissection in breast cancer. N Engl J Med 2003;349:546-553.

41) Mansel RE, Fallowfield L, Kissin M, et al. Randomized multicenter trial of sentinel node biopsy versus standard axillary treatment in operable breast cancer: the ALMANAC Trial. J Natl Cancer Inst 2006;98:599-609.

42) Symeonidis D, Koukoulis G, Tepetes K. Sentinel node navigation surgery in gastric cancer: Current status. World J Gastrointest Surg 2014;6:88-93.

43) Arigami $T$, Uenosono $Y$, Yanagita $S$, et al. Clinical application and outcomes of sentinel node navigation surgery in patients with early gastric cancer. Oncotarget 2017;8:75607-75616.

44) Park JY, Ryu KW, Eom BW, et al. Proposal of the surgical options for primary tumor control during sentinel node navigation surgery based on the discrepancy between preoperative and postoperative early gastric cancer diagnoses. Ann Surg Oncol 2014;21:11231129.

45) Kitagawa Y, Takeuchi H, Takagi Y, et al. Sentinel node mapping for gastric cancer: a prospective multicenter trial in Japan. J Clin Oncol 2013;31:3704-3710.

46) Ko WJ, Song GW, Kim WH, Hong SP, Cho JY. Endoscopic resection of early gastric cancer: current status and new approaches. Transl Gastroenterol Hepatol 2016;1:24.

47) Facciorusso A, Antonino M, Di Maso M, Muscatiello N. Endoscopic submucosal dissection vs endoscopic mucosal resection for early gastric cancer: A meta-analysis. World J Gastrointest Endosc 2014;6:555-563.

48) Hahn KY, Park CH, Lee YK, et al. Comparative study between endoscopic submucosal dissection and surgery in patients with early gastric cancer. Surg Endosc 2018;32:73-86.

49) Goto O, Takeuchi H, Kitagawa Y, Yahagi N. Hybrid surgery for early gastric cancer. Transl Gastroenterol Hepatol 2016;1:26.

50) Abe N, Mori T, Takeuchi H, et al. Successful treatment of early stage gastric cancer by laparoscopy-assisted endoscopic fullthickness resection with lymphadenectomy. Gastrointest Endosc 2008;68:1220-1224. 